6. H. A. Bethe and E. Teller, Deviations from thermal equilibrium in shock waves, BRL Rep. No. $\mathrm{X}$ 117, Aberdeen Proving Ground, Maryland.

7. S. Goldstein, Modern developments in fluid dynamics, vol. 2, Oxford University Press, 1948, p. 606. (1942).

8. H. J. Stewart, The energy equation for a viscous compressible fluid, Proc. Nat. Acad. Sci. 29, 161

9. H. Lamb, Hydrodynamics, 6th ed. Cambridge University Press, London, 1932, p. 650.

\title{
REMARKS ON THE MOTION OF ANCHOR CHAINS*
}

By R. GRAN OLSSON (Institute of Technology, Trondheim, Norway)

1. Introduction. The problem of the motion along a smooth, inclined plane of an anchor to which the end of a chain is attached (Fig. 1) has been treated in the book of S. Timoshenko and D. H. Young [1]. $\dagger$ The equation of motion is, however, given in

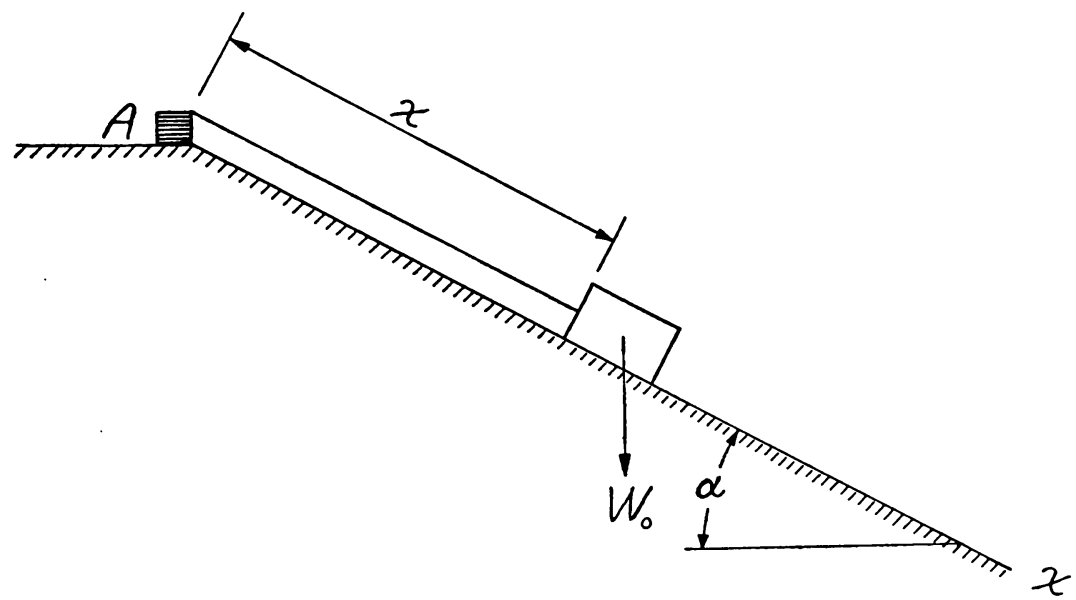

Fig. 1.

such a form that only a relation between the velocity and the displacement is obtained. In this paper it will be shown that very simple relations between the time and the kinematic quantities (displacement, velocity and acceleration) can be stated by introducing the elliptic functions of $\mathrm{K}$. Weierstrass. Very simple expressions for the kinetic quantities (momentum, kinetic and potential energy) can also be established. The motion is assumed to be frictionless, but it is not difficult to take into account a dissipative force, which is either constant or proportional to the moving mass.

2. Nomenclature. In this paper the same notations as those of Timoshenko and Young will be used: $a=$ acceleration of the weight $W_{0}\left[\mathrm{~cm} \mathrm{sec}^{-2}\right] ; B=$ rectilinear momentum [g sec]; $C=$ constant of integration; $g=$ acceleration of gravity [ $\mathrm{cm} \mathrm{sec}^{-2}$ ]; $m=$ mass of the system in motion $\left[\mathrm{g} \mathrm{cm}^{-1} \mathrm{sec}^{2}\right] ; P=$ force $[\mathrm{g}] ; q=$ weight per unit length of the chain $\left[\mathrm{g} \mathrm{cm}^{-1}\right] ; Q=$ loss of energy by percussion [g cm]; $t=$ time [sec]; $T=$ kinetic energy [g cm]; $u=$ parameter of the elliptic functions; $v=$ velocity $\left[\mathrm{cm} \sec ^{-1}\right] ; V=$

*Received May 2, 1949.

$\nmid$ Numbers in square brackets refer to the bibliography at the end of this note. 
potential energy [g cm]; $W_{0}=$ weight of the anchor [g]; $W=\left(W_{0}+q x\right)=$ weight of the anchor and a length $x$ of the chain $[\mathrm{g}] ; x=$ displacement $[\mathrm{cm}] ; x_{0}=W_{0} / q=$ length of the chain, corresponding to the weight $W_{0}[\mathrm{~cm}] ; \alpha=$ angle of the inclined plane.

Further the following abbreviations are used: $s=\left(W_{0}+q x\right) / W_{0} 4^{1 / 3} ; \xi=s 4^{1 / 3}=$ $\left(W_{0}+q x\right) / W_{0} ; \mu=(q / g) d x=$ mass of the chain element $d x\left[\mathrm{~g} \mathrm{~cm}^{-1} \sec ^{2}\right] ; \mathfrak{p}(u)=$ the elliptic $\mathfrak{p}$-function of Weierstrass; $\zeta(u)=$ the elliptic $\zeta$-function of Weierstrass.

3. Equation of motion and its integration. By using the second law of Newton in its original form,

$$
\frac{d}{d t}(m \bar{v})=\bar{P}
$$

and introducing the quantities

$$
\begin{gathered}
m=\frac{1}{g}\left(W_{0}+q x\right), \\
P=W \sin \alpha=\left(W_{0}+q x\right) \sin \alpha,
\end{gathered}
$$

we obtain

$$
\frac{1}{g} \frac{d}{d t}(W v)=W \sin \alpha
$$

After multiplying by $g d t$, we find that

$$
d(W v)=W g \sin \alpha d t,
$$

corresponding to Eq. (82c) of Timoshenko and Young [1, p. 114].

To integrate Eq. (2a), we multiply both sides by $W v$ and substitute on the righthand side, $W_{0}+q x$ for $W$ and $d x / d t$ for $v$. In this way, we obtain:

$$
W v d(W v)=\left(W_{0}+q x\right)^{2} g \sin \alpha d x .
$$

Integrating Eq. (2b), we find that

$$
\frac{1}{2}(W v)^{2}=\frac{g}{3 q}\left(W_{0}+q x\right)^{3} \sin \alpha+C .
$$

Let us assume now that when $t=0$, the body is at rest at the top of the incline. For this initial condition, the constant of integration $C$ in Eq. (3) becomes

$$
C=-\frac{g}{3 q} W_{0}^{3} \sin \alpha,
$$

and we obtain

$$
v^{2}=\frac{2 g}{3 q} \frac{\left(W_{0}+q x\right)^{3}-W_{0}^{3}}{\left(W_{0}+q x\right)^{2}} \sin \alpha .
$$

From this equation we can find the velocity $v$ for any position, provided $W_{0}$ and $q$ are known. Taking $q x$ as small compared with $W_{0}$, we find that the expression (4) reduces to

$$
v^{2} \approx 2 g x \sin \alpha,
$$

as for a body of constant mass [1, p. 115]. 
4. Introduction of the elliptic functions of Weierstrass. Substituting

$$
\begin{aligned}
& \xi=\left(W_{0}+q x\right) / W_{0}, \\
& v=\frac{d x}{d t}=\frac{W_{0}}{q} \frac{d \xi}{d t}
\end{aligned}
$$

we obtain from Eq. (4) that

$$
\left(\frac{d \xi}{d t}\right)^{2}=\frac{2}{3} \frac{g q}{W_{0}} \sin \alpha \frac{\xi^{3}-1}{\xi^{2}} .
$$

Integration of this equation gives

$$
t=\frac{\left(3 W_{0}\right)^{1 / 2}}{(2 g q \sin \alpha)^{1 / 2}} \int_{\xi_{0}}^{\xi} \frac{\xi d \xi}{\left(\xi^{3}-1\right)^{1 / 2}},
$$

where $\xi_{0}=1$, corresponding to $x=0$.

A further substitution,

$$
\xi=4^{1 / 3} s,
$$

transforms Eq. (4b) into the following integral:

$$
t=\frac{\left(6 W_{0}\right)^{1 / 2} 4^{1 / 3}}{(g q \sin \alpha)^{1 / 2}} \int_{s_{0}}^{s} \frac{s d s}{\left(4 s^{3}-1\right)^{1 / 2}} .
$$

Following Weierstrass, we introduce the elliptic integral [2]

$$
u=\int_{s}^{\infty} \frac{d s}{\left(4 s^{3}-g s_{2}-g_{3}\right)^{1 / 2}},
$$

where $g_{2}$ and $g_{3}$ are called the invariants of the doubly periodic function (the so-called p-function)

$$
s=\mathfrak{p}(u),
$$

$\mathfrak{p}(u)$ being the inverse function of the elliptic integral (7). By means of (7a) we can express the time $t$ in the following way:

$$
t=\frac{\left(6 W_{0}\right)^{1 / 2} 4^{1 / 3}}{(g q \sin \alpha)^{1 / 2}}\left\{\int_{\delta_{0}}^{\infty} \mathfrak{p}(u) d u-\int_{0}^{\infty} \mathfrak{p}(u) d u\right\}
$$

further, by introducing the $\zeta$-function of Weierstrass defined by [2, p. 183]

$$
\zeta(u)=-\int \mathfrak{p}(u) d u,
$$

we obtain $\left(x_{0}=W_{0} / q\right)$

$$
t=\frac{3.086}{(\sin \alpha)^{1 / 2}}\left(\frac{x_{0}}{g}\right)^{1 / 2}\left[\zeta(u)-\zeta\left(u_{0}\right)\right]
$$

The connection between the parameter $u$ and the displacement $x$ is given by Eqs. (5), (6) and (7a):

$$
x=\frac{W_{0}}{q}\left[4^{1 / 3} \mathfrak{p}(u)-1\right]=x_{0}\left[4^{1 / 3} \mathfrak{p}(u)-1\right]
$$


Thus we have a description of the time $t$ and the displacement $x$ given by the same parameter $u$.

The square of the velocity can now be calculated from Eq. (4):

$$
v^{2}=\frac{2 g}{3 q} W_{0} \sin \alpha \frac{4 \mathfrak{p}(u)^{3}-1}{4^{2 / 3} \mathfrak{p}(u)^{2}}=\frac{4^{1 / 3} g W_{0}}{6 q} \sin \alpha \frac{\mathfrak{p}^{\prime}(u)^{2}}{\mathfrak{p}(u)^{2}} ;
$$

the velocity itself is

$$
v=\frac{4^{1 / 6}}{6^{1 / 2}}\left(g x_{0} \sin \alpha\right)^{1 / 2} \frac{\mathfrak{p}^{\prime}(u)}{\mathfrak{p}(u)}=0 . \dot{5} 143\left(g x_{0} \sin \alpha\right)^{1 / 2} \frac{\mathfrak{p}^{\prime}(u)}{\mathfrak{p}(u)} .
$$

The acceleration can be calculated from Eq. (2), which after differentiating with respect to $t$, yields

$$
\frac{d W}{d t} v+W \frac{d v}{d t}=g W \sin \alpha
$$

Recognizing that

$$
v \frac{d W}{d t}=q\left(\frac{d x}{d t}\right)^{2}=q v^{2},
$$

we obtain the acceleration from Eq. (8):

$$
a=\frac{d v}{d t}=g \sin \alpha-\frac{q}{W} v=g \sin \alpha\left[1-\frac{q x_{0}}{W_{0}+q x} \frac{4^{1 / 3}}{6} \sin \alpha \frac{\mathfrak{p}^{\prime}(u)^{2}}{\mathfrak{p}(u)^{2}}\right] .
$$

We observe that [see Eqs. (5), (6) and (7a)]

$$
W_{0}+q x=W_{0} \xi=W_{0} 4^{1 / 3} s=W_{0} 4^{1 / 3} \mathfrak{p}(u) ;
$$

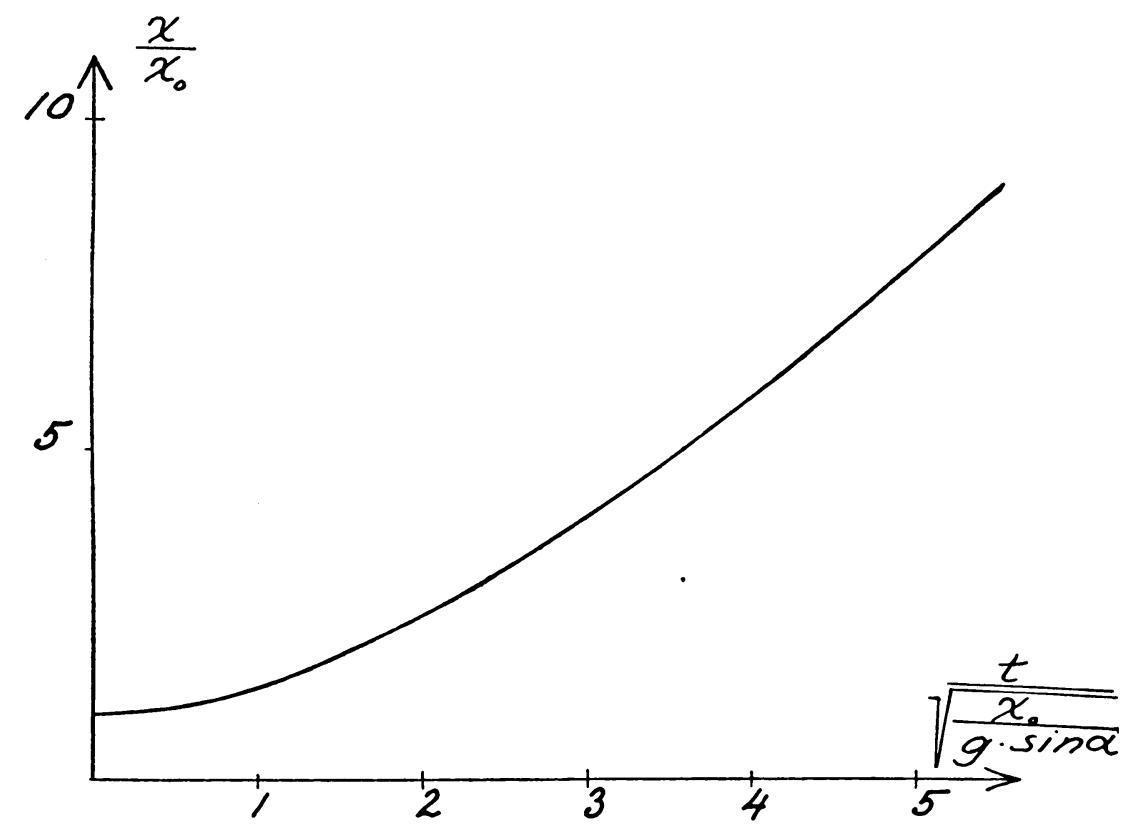

Fig. 2. 
hence the final expression for the acceleration is

$$
a=g \sin \alpha\left[1-\frac{1}{6} \frac{\mathfrak{p}^{\prime}(u)^{2}}{\mathfrak{p}(u)^{3}}\right]=\frac{g}{6} \sin \alpha\left[2+\mathfrak{p}(u)^{-3}\right] .
$$

In the last expression we have used the relation between the p-function and its derivative $\mathfrak{p}^{\prime}(u)[2$, p. 182$]$, viz.,

$$
\mathfrak{p}^{\prime}(u)^{2}=4 \mathfrak{p}(u)^{3}-1 .
$$

Comparing Eqs. (4b) and (7) we see that in the present problem the invariant $g_{2}$ is equal to zero; for this case the $\mathfrak{p}$ - and $\zeta$-functions are tabulated by A. G. Greenhill [3]. Thus we can calculate the diagrams for displacement, velocity and acceleration depending on time $t$; they are presented in Figs. 2, 3 and 4, respectively. It can easily be proved

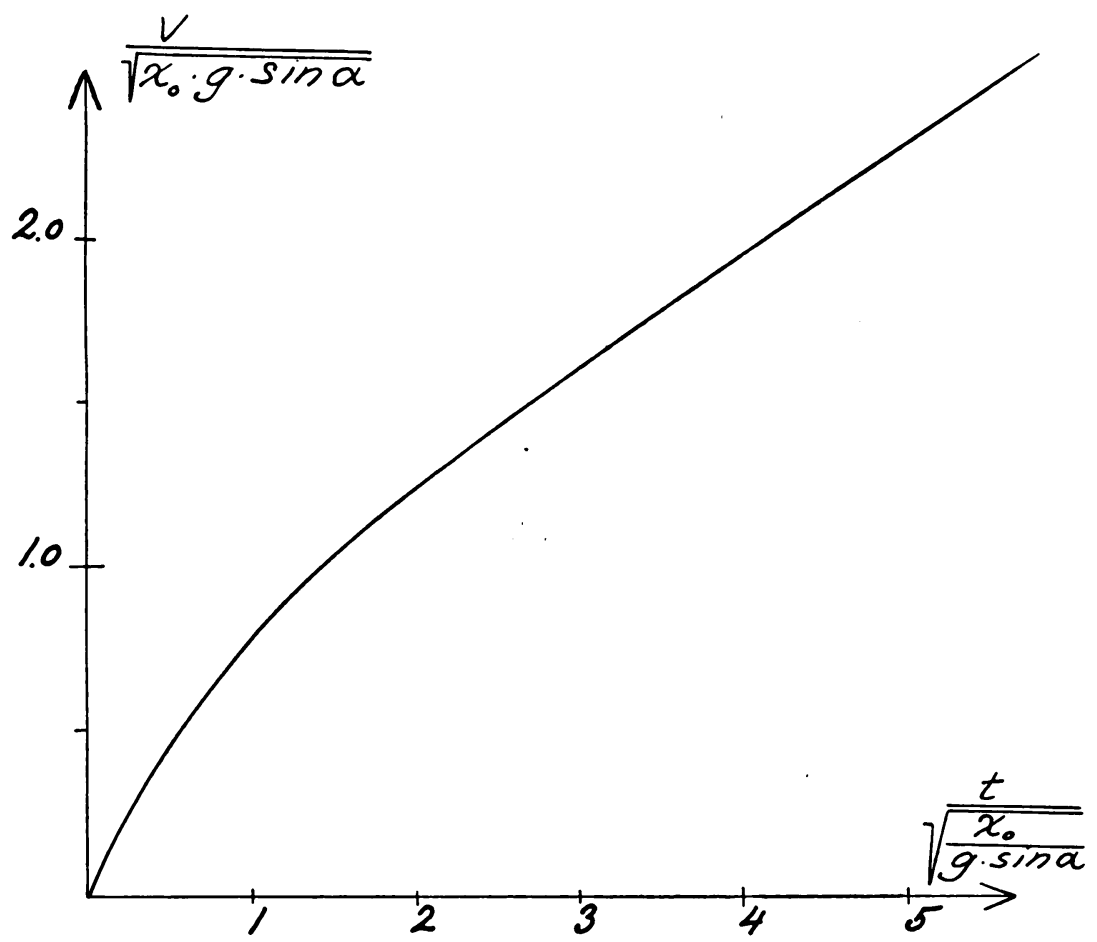

FIG. 3.

that the curve in Fig. 4 has a horizontal tangent at $t=0$ and a point of inflection for $\mathfrak{p}(u)=(5 / 14)^{1 / 3}=0.710$, corresponding to $u=1.275$.

There is an asymptotic value of acceleration equal to $g / 3$, and this is obtained practically with $u$ approximately equal to 0.5 . The constant value of acceleration corresponds to a straight line for velocity and a parabolic curve for displacement.

5. Presentation of the kinetic quantities. Besides the kinematic quantities, it is interesting to present the kinetic quantities-momentum and energy. 
For the momentum we have:

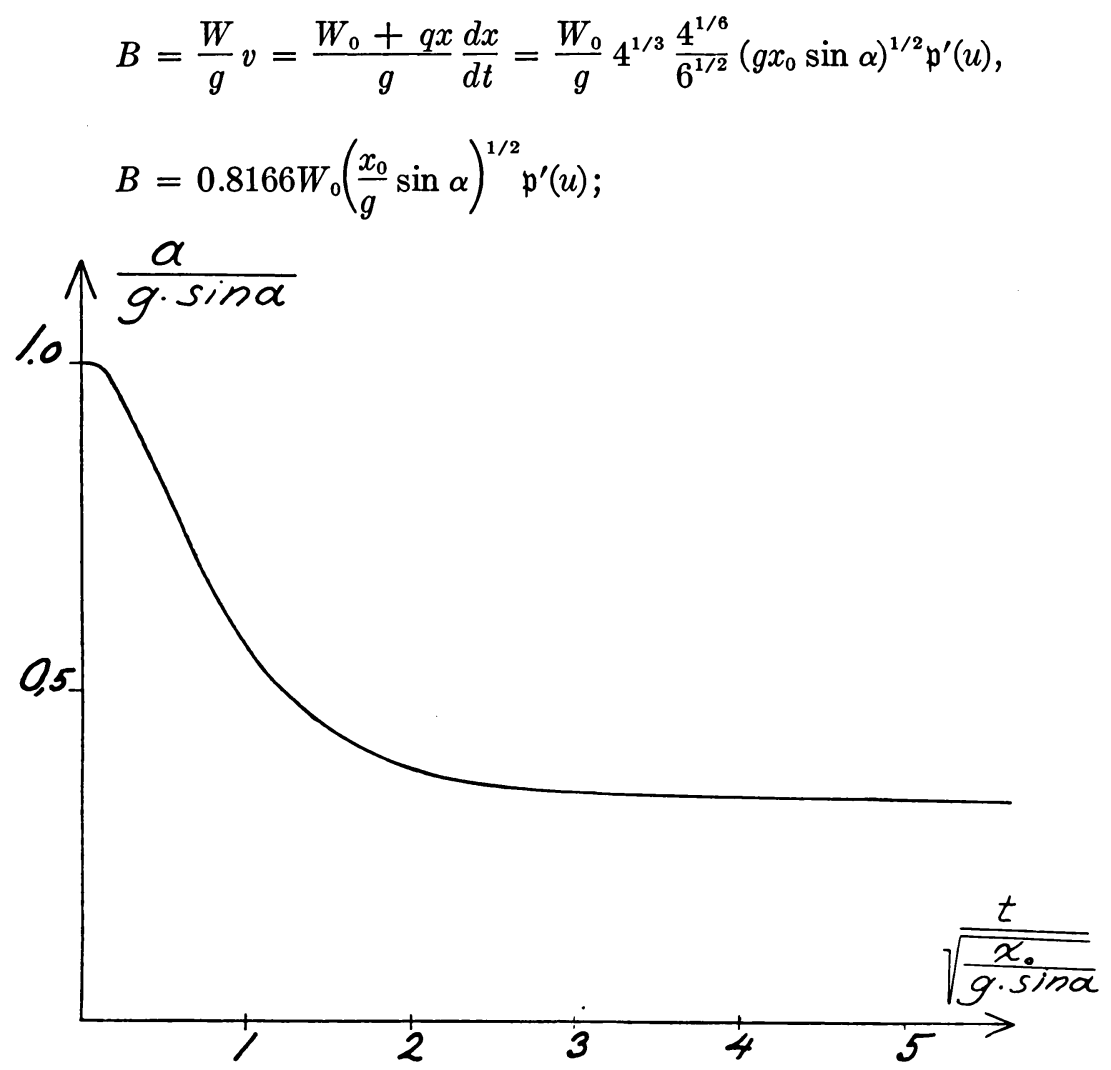

Fig. 4.

and for the kinetic energy:

$$
T=\frac{W}{2 g} v^{2}=\frac{W_{0}+q x}{12 q} 4^{1 / 3} W_{0} \sin \alpha \frac{\mathfrak{p}^{\prime}(u)^{2}}{\mathfrak{p}(u)^{2}}=\frac{2^{1 / 3}}{6} \frac{W_{0}^{2}}{q} \sin \alpha \frac{\mathfrak{p}^{\prime}(u)^{2}}{\mathfrak{p}(u)} .
$$

Introducing $\mathfrak{p}^{\prime}(u)$ expressed by $\mathfrak{p}(u)$ (Eq. (9)), we obtain

$$
T=0.210 \frac{W_{0}^{2}}{q} \sin \alpha\left[4 \mathfrak{p}(u)^{2}-\mathfrak{p}(u)^{-1}\right],
$$

and for large values of the parameter $u$,

$$
T=0.840 W_{0} x_{0} \sin \alpha \mathfrak{p}(u)^{2} .
$$

Finally, the potential energy

$$
V=-\left(W_{0} x+q x \frac{x}{2}\right) \sin \alpha=-x \sin \alpha\left(W_{0}+\frac{q}{2} x\right) .
$$

Inserting the expression for the displacement $x$ (Eq. (5a)), we find that

$$
V=-\frac{W_{0} x_{0}}{2} \sin \alpha\left[22^{1 / 3} \mathfrak{p}(u)^{2}-1\right] .
$$


6. The transformation of energy of the system. It can be shown that the theorem of conservation of energy in the usual form does not hold in the present problem. To the energy equation we must add a term, involving the loss of energy by percussion of the links of the chain. The calculation has the following form.

The potential energy is obtained by integration,

$$
V=W_{0} \sin \alpha\left(x_{0}-x\right)+\frac{q}{2} \sin \alpha\left(x_{0}^{2}-x^{2}\right),
$$

and, as above, the kinetic energy is

$$
T=\frac{m}{2} v^{2}=\frac{1}{2 g}\left(W_{0}+q x\right) v^{2} .
$$

Differentiating with respect to time yields

$$
\begin{gathered}
\frac{d V}{d t}=-\left(W_{0}+q x\right) \sin \alpha \frac{d x}{d t}, \\
\frac{d T}{d t}=\frac{v}{2 g}\left[2\left(W_{0}+q x\right) \frac{d v}{d t}+q v^{2}\right],
\end{gathered}
$$

and after addition,

$$
\frac{d V}{d t}+\frac{d T}{d t}=-\left(W_{0}+q x\right) \sin \alpha v+\frac{v}{2 g}\left[2\left(W_{0}+q x\right) \frac{d v}{d t}+q v^{2}\right] .
$$

Equation (2) may be written as

$$
\frac{1}{g}\left(W_{0}+q x\right) \frac{d v}{d t}+\frac{q}{g} v^{2}-\left(W_{0}+q x\right) \sin \alpha=0 .
$$

If we add the term

$$
\frac{d Q}{d t}=\frac{q}{2 g} v^{3}
$$

to Eq. (15), we obtain

$$
\frac{d V}{d t}+\frac{d T}{d t}+\frac{d Q}{d t}=v\left[\frac{1}{g}\left(W_{0}+q x\right) \frac{d v}{d t}+\frac{q}{g} v^{2}-\left(W_{0}+q x\right) \sin \alpha\right],
$$

where the terms in the brackets correspond to Eq. (2c). Thus Eq. (15a) may be written as

$$
\frac{d V}{d t}+\frac{d T}{d t}+\frac{d Q}{d t}=0
$$

or, after integration,

$$
V+T+Q=\text { const. }
$$

The quantity $d Q$ may be written as

$$
d Q=\frac{q}{2 g} v^{2} d x
$$


and interpreted as the loss of energy by non-elastic percussion of the links of the chain. If we write

$$
\mu=\frac{q}{g} d x
$$

where $\mu$ indicates the mass of the chain element $d x$ and

$$
d Q=\frac{\mu}{2} v^{2}
$$

$\mu$ is also called the reduced mass by percussion of the chain elements (Carnot).

\section{REFERENCES}

(1) S. Timoshenko and D. H. Young, Advanced dynamics, McGraw-Hill Book Co., Inc., New York, 1948, pp. 114-116.

(2) P. Frank and R. v. Mises, Die Differential- und Integralgleichungen der Mechanik und Physik, 2. ed., M. S. Rosenberg, New York, 1943, p. 180.

(3) A. G. Greenhill, Proc. Roy. Artillery Inst. 17, 181-226 (1889); or E. Jahnke and F. Emde, Tables of functions, Dover Publications, New York, 1945, pp. 102-104. (The value of $\zeta(u)=3.7174$ for $r=31^{\circ}$ is incorrect. A better value is $\zeta=3.7952$.)

\section{A NOTE ON SUPERSONIC FLOW IN THE TREFFTZ PLANE*}

By JOHN W. MILES (University of California, Los Angeles)

Introduction-The general problem of linearized, supersonic flow has been treated from the standpoint of Fourier transforms in an earlier paper. ${ }^{1}$ The methods set forth therein will be used to calculate the flow conditions far downstream from a lifting surface. If $x$ is the streamwise coordinate, cf. Fig. 1, the downstream plane $x=\infty$ is designated as the Trefftz plane. ${ }^{2}$

This same problem has been treated by Lagerstrom and Graham, ${ }^{3}$ who took advantage of the fact that the flow in the Trefftz plane satisfies Laplace's equation. These same authors also made calculations in regions not infinitely removed from the wing by making use of conical flow methods.

Statement of problem-The vector perturbation velocity due to the presence of the lifting surface is given by

$$
\mathbf{q}(x, y, z)=U \nabla \phi(x, y, z),
$$

where $\phi$ satisfies the linearized equation

$$
\phi_{x x}=\phi_{y y}+\phi_{z z}
$$

${ }^{*}$ Received Feb. 11, 1949.

1J. W. Miles, On linearized supersonic airfoil theory, No. Amer. Avia. Rep. AL-801, Nov., 1948.

${ }^{2}$ E. Trefftz, Zur Prandtlschen Tragflaechentheorie, Math. Ann. 82, 306-319 (1921).

${ }^{3}$ P. A. Lagerstrom and M. E. Graham, Downwash and sidewash induced by three dimensional lifting wings in supersonic flow, Douglas Aircraft Rep. SM-13007, April, 1947. 